\title{
Loss analysis in luminescent sheet concentrators: from ideal to real system
}

\author{
Florian Proise $^{1,3}$, Anne-Laure Joudrier ${ }^{2}$, Jean-Luc Pelouard $^{3}$, and Jean-François Guillemoles ${ }^{1, *}$ \\ ${ }^{1}$ CNRS, Institut Photovoltaïque d'Ile de France IPVF-UMR 9006, 30 Route Départementale 128, 91120 Palaiseau, France \\ 2 ENSCP Chimie-Paristech, Institut Photovoltaïque d'Ile de France IPVF-UMR 9006, 30 Route Départementale 128, \\ 91120 Palaiseau, France \\ ${ }^{3}$ Centre de Nanosciences et Nanotechnologies C2N, Route de Nozay, 91460 Marcoussis, France
}

Received: 21 December 2016 / Received in final form: 15 October 2018 / Accepted: 12 November 2018

\begin{abstract}
Thermodynamic limits of luminescent sheet concentrators (LSC) are three orders of magnitude higher than record ones made up to now. This paper aims at understanding why there is such a gap and what is the link between ideal and real LSC. Computational modeling enables to evaluate separately the different loss mechanisms, to determine their respective weight, and to correlate them to accessible physical parameters, such as geometrical ratio, photoluminescence quantum yield, etc. From an ideal system, the different parameters have been degraded to obtain a real system and the performances of each system have been simulated. The high interdependency of different loss mechanisms has also been studied in several cases. In a second part, more realistic cases are addressed to show how performances is impacted by non-idealities and where does the principal limitations come from. Practically achievable efficiencies are suggested, based on state-of-the-art technologies and material properties. Finally, promising directions for the search of better systems are proposed.
\end{abstract}

Keywords: luminescent / concentrator / Monte Carlo / photovoltaic

\section{Introduction}

Light concentration photovoltaic systems (CPV) have been developed in the past decades along with the increasing importance of light management in the improvement of photovoltaic devices. The purpose of concentration is to achieve cost reduction through semiconductor material saving and efficiency enhancement due to higher light concentration. Most concentrating systems use lenses and mirrors to concentrate the direct sunlight hundreds of times (typically X400-X700) on solar cells $[1,2]$. Counterparts of high-concentration photovoltaics (HCPV) are the need of a sun-tracking system and the loss of diffused sunlight, which make these concentrating systems suitable only for sunny regions with efficient solar cells. On the contrary, low-concentration photovoltaics (LCPV) have also been developed. Being mainly non-imaging, they are cheaper and may concentrate both direct and diffuse sunlight, do not require tracking, and, consequently, have a different scope of application than HCPV.

\footnotetext{
* e-mail: jf.guillemoles@chimie-paristech.fr
}

Luminescent solar concentrator (LSC) is a popular example of LCPV, studied since the early 1980s [3] and being now subject of numerous researches [4,5]. The principle of LSC is to trap light inside a dielectric matrix doped with organic or inorganic dyes until it reaches the cells. A part of the sunlight is absorbed by dye particles and isotropically emitted, allowing a portion of light to be trapped by total internal reflection. The dye absorption/ emission process generates entropy through heat, which allows going beyond the scope of conservation [6] and thus makes concentration factor higher than the classical limit possible.

Theoretically, this concept is appealing because concentration factors up to 1000 without tracking are possible [7]. Practically, experimental performances fall far from this expectation [4,8]. Sloof et al. obtained the world record of solar-to-electric conversion efficiency per unit collector surface of $7.1 \%$ [8] and many other research teams reached efficiency around $3 \%$ to $6.7 \%$ [9-13]. However, the common point of record LSC systems is their small sizes, which indicates that losses are highly dependent on the optical path.

In this paper, we explain why there is such a gap between theory and experiment analyzing the different loss 
mechanisms by Monte Carlo simulations. Theoretical work has been done either on ideal or realistic systems via raytracing or thermodynamics-based modeling $[14,15]$, but the link between these two cases has never been studied until now.

The first part aims at introducing the problematic and validating our code finding the same asymptotical response of an LSC with an idealized dye compared to thermodynamic limits. Then, different loss mechanisms are analyzed degrading some parameters of this all-ideal system. First, three LSC parameters are studied separately to highlight loss hierarchy. Then two of them are degraded simultaneously to investigate interdependencies. To our knowledge, it is the first time that interdependences between loss mechanisms are addressed. We found these to be especially based on PLQY for non-radiative loss, and $R_{\text {back }}$ for back loss. Furthermore, we move from an all-ideal system to a realistic one through an intermediate dye to highlight the shift between these two extreme cases. Finally, we study expected performances, losses distribution as well as some pathways for improvements.

\section{Method}

In this section we describe first the model system and then the Monte Carlo computational method.

\subsection{Description of the LSC}

Loss mechanisms are studied on a model rectangular LSC. PV cells pave the reflective back surface (bottom-mounted configuration) with a coverage fraction $f$ (total PV cell area divided by total area). Homogeneous PV cell repartition at the rear surface avoids scaling effects compared to configurations in which PV cells are on the concentrator edges, although these two configurations have almost the same potential for conversion efficiency [16]. In such a system five basic loss mechanisms exist, which are depicted in Figure 1.

We used a typical case for application, a cavity consisting of a transparent matrix (ideal transmission $T=1$ for all wavelengths) of refractive index 1.5 (close to polymethyl methacrylate, a widely used polymer for LSC) and a thickness $d$ of $0.5 \mathrm{~cm}$.

The host matrix is doped with a dye characterized by its photoluminescence quantum yield (PLQY) which is not necessarily close to unity. Three dyes (ideal, semi-ideal, and realistic) were considered in this work, but only the ideal dye and the real dye are presented here in detail. The commercial dye we choose as reference real dye in this study is Lumogen Red 305 (R305), a standard fluorophore commonly used for LSC applications because of its high PLQY.

The ideal dye enables to simulate LSC system as close as possible to thermodynamic limits, R305 enables to assess what can be expected in practice, whereas the semi-ideal dye highlights how the transition between these two extreme cases occurs and helps illustrate the desirable characteristics to search for improved dyes. The semi-ideal dye absorption spectrum has the same absorption and emission shape as the ideal dye, but is modified to better match R305 in terms of absorption edge and Stokes shift.

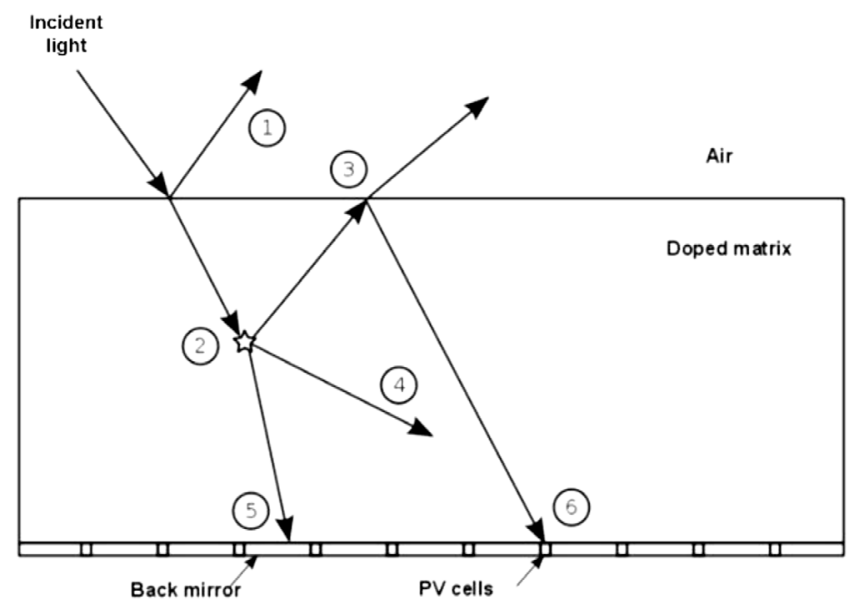

Fig. 1. Schematic of the LSC used to perform modeling and loss mechanisms. 1: external losses; 2-5: internal losses. 2: dye absorption and non-radiative de-excitation, 3: non-trapping at the front surface, 4: absorption by the host matrix, 5: mirror absorption, 6: collected photons.

The results on semi-ideal dye will not be presented in detail, as it is only used for illustrative purposes.

The absorption coefficient of the ideal dye $\alpha_{\text {dye }}$ is taken constant over two ranges of the spectrum, $\alpha_{\text {dye }}=3 / d$ for $\lambda<\lambda_{\mathrm{c}}(95 \%$ absorption probability on a distance $d)$ and $\alpha_{\text {dye }}=0.03 / d$ for $\lambda_{\mathrm{c}}<\lambda<\lambda_{\mathrm{g}}$, where $\lambda_{\mathrm{c}}$ is the cutoff wavelength and $\lambda_{\mathrm{g}}$ is the solar cell gap wavelength. This corresponds to a good balance between absorption of incoming light and self-absorption of luminescent light, and was also used in [7]. The emission spectrum is deduced from Kirchhoff's law [17]:

$$
e(E) \propto n_{m a t} \alpha(E) E^{2} e^{-\frac{E}{k T}}
$$

where $e$ is the emission coefficient, $E$ is the photon energy, $n_{\text {mat }}$ is the matrix refractive index, $\alpha(E)$ is the absorption coefficient, $k$ is the Boltzmann constant, and $T$ is the temperature.

In addition, the use of a photonic stop-band (PSB) at the front interface may improve the light trapping inside the LSC (Fig. 2) [18]. Its role is to transmit light absorbable by the dye $\left(T=1\right.$ and $R=0$ for $\lambda<\lambda_{\mathrm{c}}$ ) and to reflect the emitted light ( $T=0$ and $R=1$ for $\lambda_{\mathrm{c}}<\lambda<\lambda_{\mathrm{g}}$ ) (Fig. 3). Figure 3 shows the absorption and emission spectra for the ideal and R305 dye. It is known that the reflection spectra change as a function of the incidence angle, but in a first approach and for the modeling, we consider that PSB has a reflection index $R=1$ for $\lambda_{\mathrm{c}}<\lambda<\lambda_{\mathrm{g}}$ and for all angles $\theta$. For the modeling, we consider opal photonic crystal properties.

In this paper, the PV cells placed at the rear surface have an ideal external quantum efficiency and a bandgap of $1.55 \mathrm{eV}(800 \mathrm{~nm})$ is chosen to match R305. For example, an AlGaAs solar cell with $8 \% \mathrm{Al}$, would have a suitable bandgap.

The non-radiative dark current $J_{0}$ of the $\mathrm{PV}$ cells is set at $10^{-12} \mathrm{~mA} \cdot \mathrm{cm}^{-1}$ for the open-circuit voltage calculation. From this, it is possible to compute the efficiency of 


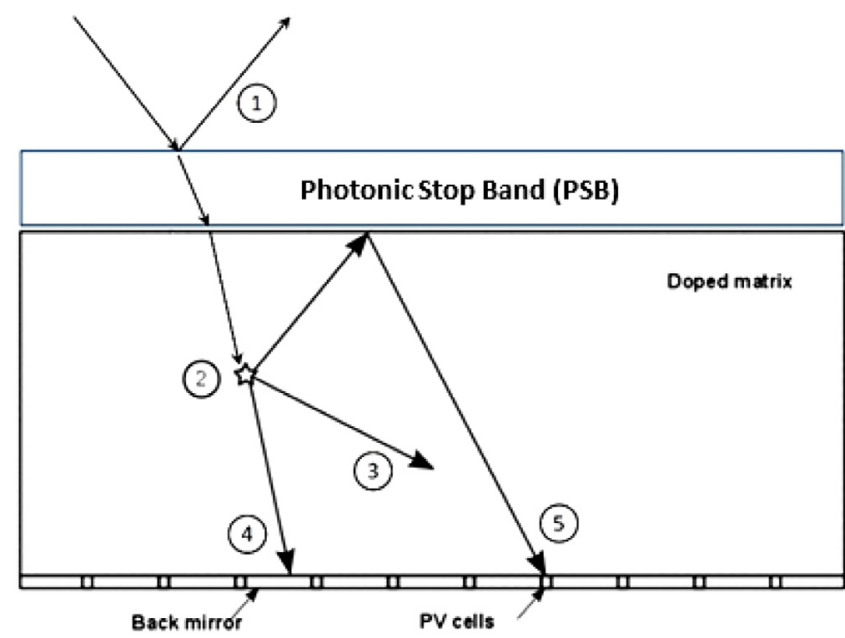

Fig. 2. Schematic of the LSC with PSB in front surface. Like in Figure 1, loss mechanisms are represented. 1: external losses, 2: dye absorption, and non-radiative de-excitation, 3 : absorption by the host matrix, 4: mirror absorption, 5: collected photons.

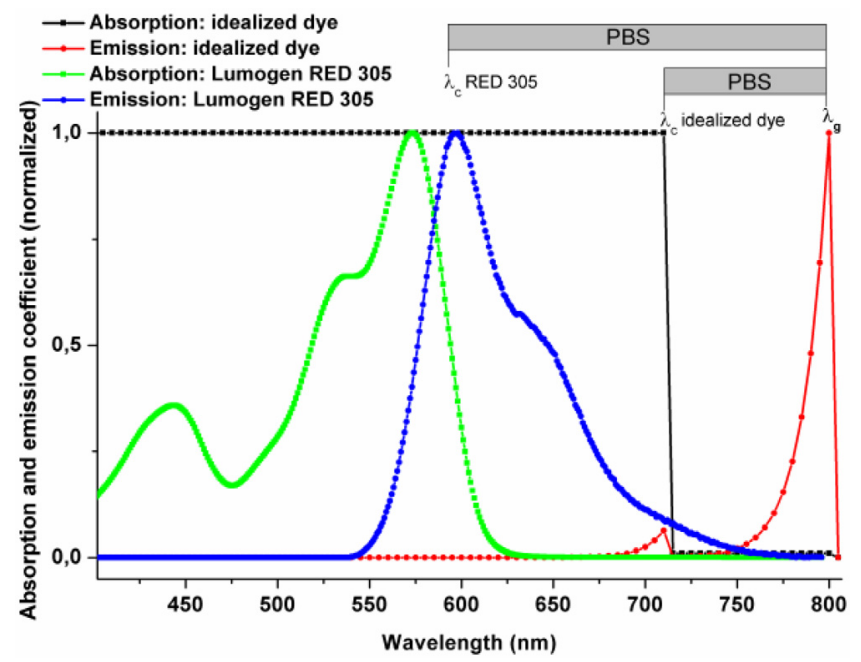

Fig. 3. Normalized absorption and emission coefficients of R305 (green and blue) and an ideal dye (black and red) with $\alpha_{\text {dye }}=3 / d$ when $0<\lambda<\lambda_{\mathrm{c}}$ and $\alpha_{\text {dye }}=0.03 / d$ when $\lambda_{\mathrm{c}}<\lambda<\lambda_{\mathrm{g}}$, with $\lambda_{\mathrm{c}}=715 \mathrm{~nm}$ ( $d$ stands for the LSC thickness). The gray rectangle noted "PSB" represents the wavelength range where the PSB reflects totally the emitted light $(T=0, R=1)$ with omnidirectional reflectance.

the system from the diode characteristics: $J=J_{0}\left(e^{\frac{q V}{k T}}-1\right)-J_{\mathrm{sc}}$ and $J_{\mathrm{sc}}$ is calculated by Monte Carlo simulations. This is used in Figure 4.

\subsection{Monte Carlo modeling}

At the beginning of the simulation, $10^{5}$ photons are impinging in parallel, at normal incidence, with an AM 1.5 solar spectrum distribution, in the range of $350-800 \mathrm{~nm}$. After testing if photons are not reflected at the front surface, their outcome in the concentrator is established,
Table 1. The four possible interaction families are listed, together with the involved part of the LSC and parameters.

\begin{tabular}{lll}
\hline Interactions & LSC constituent & $\begin{array}{l}\text { LSC parameter } \\
\text { involved }\end{array}$ \\
\hline Absorption & Matrix & $\alpha_{\text {mat }}$ \\
& Dye & $\alpha_{\text {dye }}$ \\
Emission & Dye & PLQY \\
Reflection & Front interface & $R_{\text {fresnel }}\left(+R_{\mathrm{PSB}}\right)$ \\
& Back interface & $R_{\text {reflector }}$ \\
Collection & PV cell & $f$ \\
\hline
\end{tabular}

taking into consideration possible interactions listed with the specific parameters involved in Table 1.

The model considered is 3D (three spatial dimensions). The probability of collection at the back surface by solar cells is determined by their coverage fraction $f$ (statistical method introduced in [7]).

The output is the spectral distribution of photons "fates", as a function of their wavelength, enabling to compute the optical efficiency $\eta_{\text {opt }}$ given by the ratio between the number of collected photons $N_{\text {coll }}$ over the total number of incident photons $N_{\text {tot }}: \eta_{\text {opt }}=N_{\text {coll }} / N_{\text {tot }}$. The concentration factor $c$ is then given by the optical efficiency divided by the coverage fraction $f: c=\eta_{\mathrm{opt}} / f$.

The code has been tested and validated by calculating asymptotical limits and comparing the results found with the thermodynamic limits (see Sect. 3.1).

\section{Results}

In Section 3, we present the results obtained by modeling successively an ideal and a realistic LSC system with our Monte Carlo code. The semi-ideal system was simulated too, for illustrative purposes [20].

Starting with the ideal case, we search the optimal Stokes shift as a function of the photon concentration ratio. Then we investigate the influence of the different LSC parameters and their interdependencies before moving to the realistic system.

The Stokes shift of the ideal dye, which can be defined as $E_{\text {absorption }}-E_{\text {emission }}=\frac{h c}{\lambda_{g}}-\frac{h c}{\lambda_{c}}$, has been optimized calculating the PV cell efficiency (without PSB) versus the coverage fraction (Fig. 4).

Figure 4 shows that the ideal Stokes shift depends on the coverage fraction. It is understandable considering that for a low coverage fraction, the mean optical path of photons must be high enough to reach a PV cell, resulting in a necessarily high optical efficiency and consequently a high Stokes shift.

Then for the three cases we will simulate, we have chosen a Stokes shift of $0.2 \mathrm{eV}$, which is an optimum for a coverage fraction of 0.01 . A high Stokes shift implies an efficient trapping (decreased low wavelength re-emission probability) but also important external loss (increased incoming light reflection). These two antagonist effects lead to a trade-off based upon the need for the efficient 


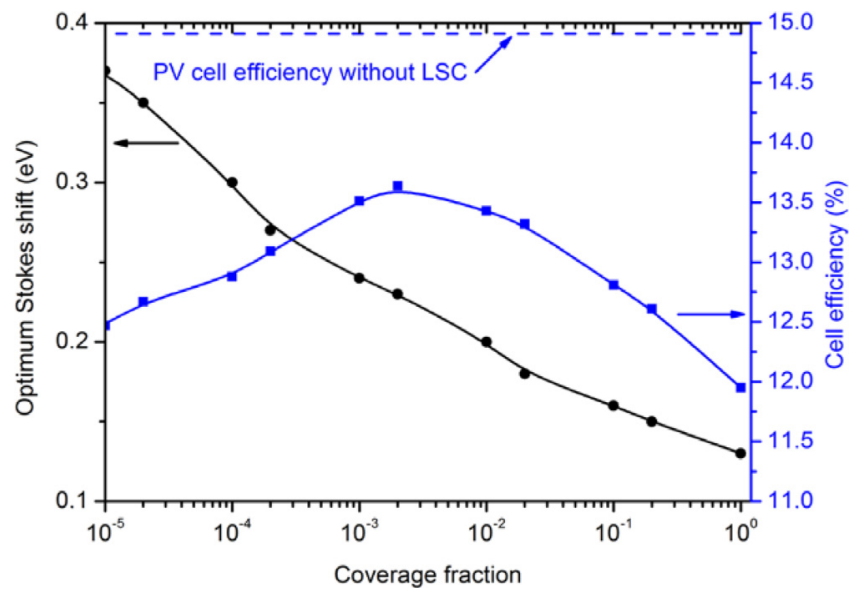

Fig. 4. Optimum Stokes shift without PSB and associated PV cell efficiency as a function of the coverage fraction. For comparison, the blue dash line represents the PV cell efficiency without LSC. Symbols (black dots, blue squares) are obtained by simulations, lines are only a guide for the eye.

trapping, defined by the photon mean optical path before reaching a PV cell, which is inversely proportional to the coverage fraction.

In this case, the only way of losing photons is front loss due to photons redirected into escaping modes because of self-absorption.

The efficiency as a function of coverage fraction at this Stokes shift is also represented for comparison in Figure 4.

\subsection{All ideal LSC}

Losses cannot be totally avoided, even in an ideal system, because of thermodynamic limits. To reproduce ideal conditions, we simulate the LSC system with the ideal dye described in Section 2, and ideal LSC parameters $\left(R_{\text {back }}=1, \mathrm{PLQY}=1, \alpha_{\mathrm{mat}}=0 \mathrm{~cm}^{-1}\right)$. The boundary condition at the edges is periodic. In this highly idealized case, the only loss channel is the external loss and the photon escape through the front surface (loss 1 and 3 in Fig. 1). Both cases, with and without PSB, are presented in Figure 5. Clearly, the optical efficiency is a monotonically increasing function of $f$, and the concentration factor is a monotonically decreasing function of $f$.

Thus, there is a fine balance to find in order to perform optimally, knowing that each loss depends on its LSC parameter (PLQY for non-radiative loss, $R_{\text {back }}$ for back loss, and so on) but also on all other parameters.

Since all parameters are correlated in a complex way, the analytical prediction of the system losses is challenging. That is why numerical simulations are necessary to understand the physics of LSC.

\subsection{From ideal to realistic LSC}

To be able to compare a realistic LSC with a commercial dye from an idealized concentrator, we adapt the ideal dye to the commercial one. The absorption spectrum is modified in order to fit the emission probability and the
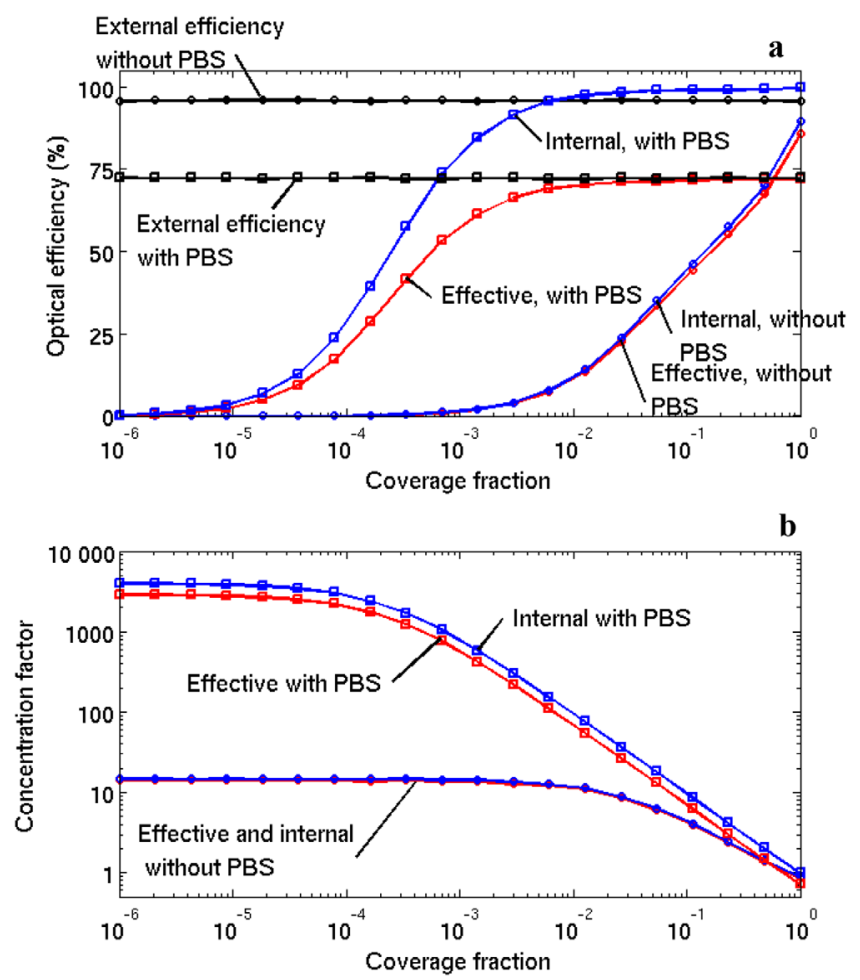

Fig. 5. (a) Dependence of optical efficiency and (b) concentration factor $c$ on the coverage fraction, for an ideal system with and without PSB, obtained by simulations with our Monte Carlo code. Internal values take into consideration only internal losses whereas effective ones account for both internal and external losses. The two horizontal lines in part (a) represent the external efficiency with and without PSB. It does not depend on the coverage fraction. Effective efficiency is the product of the internal and external efficiency.

absorption probability of R305, keeping the absorption stair shape of the ideal dye. The fit leads to a semi-ideal dye with $\alpha=3 / d$ when $0<\lambda<\lambda_{\mathrm{c}}$ and $\alpha=0.003 / d$ when $\lambda_{\mathrm{c}}<\lambda$ $<\lambda_{\mathrm{g}}$, with $\lambda_{\mathrm{c}}=615 \mathrm{~nm}$ and $\lambda_{\mathrm{g}}=675 \mathrm{~nm}$ (where $d$ is the LSC thickness) (Fig. 6).

This intermediate case exhibits a higher probability to emit in the low wavelength emission peak spectral region as compared to the ideal dye because the Stokes shift is smaller than the optimum.

This leads to two main consequences: higher PLQY losses through more reabsorption processes and less efficient PSB trapping. The maximum concentration factor achievable is 1900 against 4000 for the ideal dye, which is a direct consequence of the lower Stokes shift $(0.179<0.200 \mathrm{eV})$. This gives still an achievable high concentration ratio. These results are also consistent with Figure 4 . For $\lambda_{\mathrm{c}}<\lambda<\lambda_{\mathrm{g}}$, the overlap between absorption and emission spectra increases. There is more reabsorption by the dye which implies more losses.

The conclusion is that the Stokes shift is not the only cause for losses, and the role of the lack of abruptness of the absorption threshold must be considered [20]. This case with a semi-ideal dye is only an intermediate case which will be improved by the real dye and then to attain the real system. 


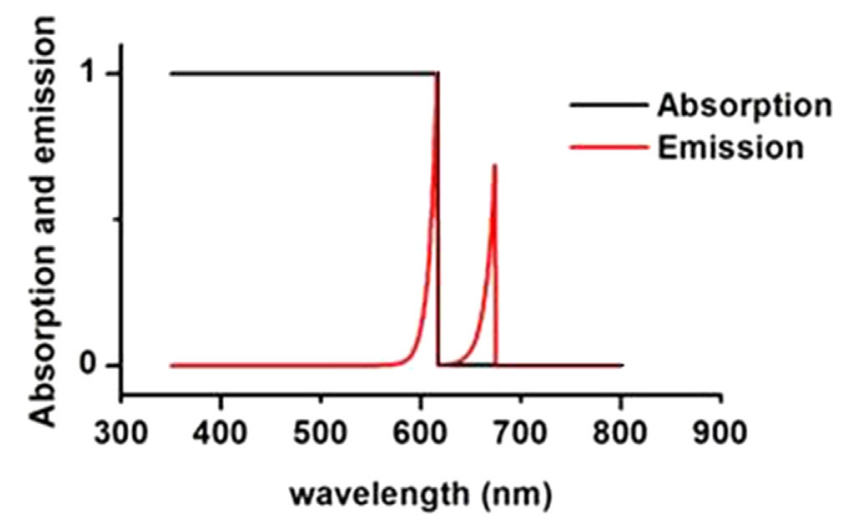

Fig. 6. Normalized absorption and emission spectra of the semiideal dye.

\subsection{Real dye}

To address the realistic LSC issue, we have modeled the real dye with the absorption and emission spectrum of R305 described in Section 2.1. The dye concentration is $270 \mathrm{ppm}$, which corresponds to a probability of 0.99 for a photon of $475 \mathrm{~nm}$ to be absorbed on two times the LSC thickness. The wavelength $475 \mathrm{~nm}$ corresponds to the valley in the RED305 absorption coefficient spectrum in Figure 3. The dye is enclosed in a polymethyl methacrylate (PMMA) matrix, which is a suitable material for this application because of its high transparency in the visible, stability, and doping facility.

Before going further, one can compare in Figure 7 the influence of $R_{\text {back }}$, PLQY, and $R_{\mathrm{PSB}}$ for the three dyes considered: the ideal, semi-ideal, and R305 dye. The PLQY is not a free parameter for the R305 dye, but it highlights the influence of the absorption and emission spectra shape.

The accident point for $R_{\mathrm{PSB}}$ in the semi-ideal case arises because the PSB trapping effect is poor and consequently even with no PSB $\left(R_{\mathrm{PSB}}=0\right)$, the drop of concentration factor cannot reach $c^{\max } / 100$. The bad trapping property is due to a higher probability of re-emission at wavelength not reflected by the $\mathrm{PSB}$. In addition, the maximum concentration factor achievable is different for the three dyes: 4000, 1900, 1400 for ideal, semi-ideal, R305 dyes, respectively. These concentration ratios are still quite high, even in the case of the R305. Without PSB, these concentration ratios are in good agreement with experimental results [21,22]. With PSB, no experimental work has been published yet.

\subsection{Real system}

In this section we introduce a real LSC with R305 dye and realistic parameters.

We address a LSC system with a PSB at the front surface. The considered PSB is a silica opal filter which reflects or transmits photons according to the reflection spectrum shown in Figure 8 adapted from [19]. For more precision, a map as a function of angle and wavelength is also given in Figure 8 from the same reference. This opal is not ideal but seems to be a reasonable example of optical

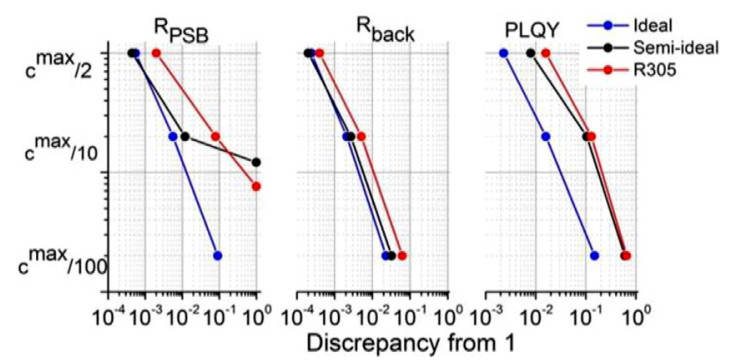

Fig. 7. Effect of $R_{\mathrm{PSB}}, R_{\text {back, }}$, and PLQY non-ideality on the concentration factor for the ideal (blue), semi-ideal (black), and real R305 (red) dye.
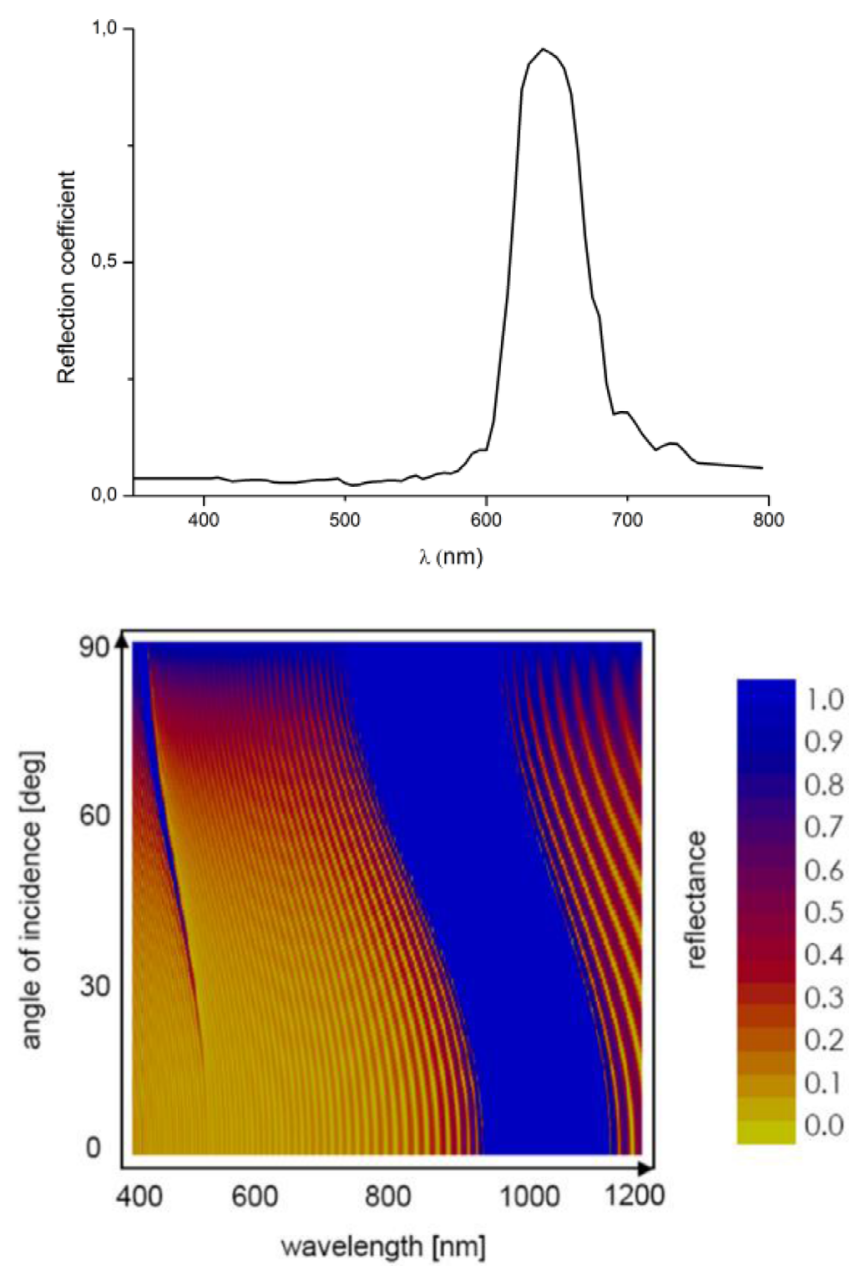

Fig. 8. Reflection coefficient of a simulated silica opal, adapted from [19]. Top: The reflectance spectrum used, with a shift of the entire plot of $50 \mathrm{~nm}$ to higher wavelengths, which corresponds to a larger sphere size. Bottom: Original figure of reference [19], showing the angular dependence.

filter which may be used with LSC. As indicated in Section 2.1, we consider no angular dependencies but only the normal incidence spectrum.

Dimensions are $5 \times 5 \times 0.5 \mathrm{~cm}^{3}$, and a silver mirror with a reflection coefficient $R_{\text {side }}$ is deposited at the edges. 


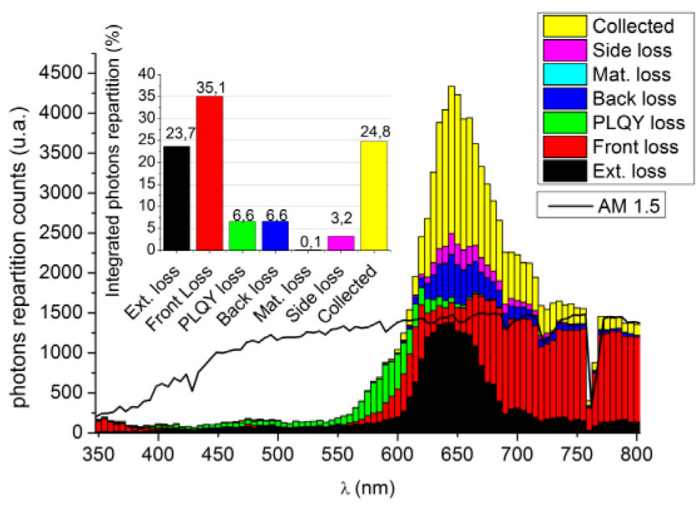

Fig. 9. Spectral repartition of photons according to the different loss mechanisms. Inset represents the integrated repartition. The LSC parameters have been set as follows: PLQY $=0.95, R_{\text {back }}=$ $R_{\text {side }}=0.97, \alpha_{\text {PMMA }}=10^{-3} \mathrm{~cm}^{-1}$ and $f=0.1$.

The dye concentration value has been optimized and worth 200 ppm to reconcile a high absorption and a low selfabsorption. Results are shown in Figure 9.

This LSC has a concentration factor of 2.48 , which is a direct consequence of a $24.8 \%$ effective optical efficiency and $f=0.1$. This is far from the effective optical efficiency of the all ideal case of $72 \%$ (see Fig. 5). This is mainly due to high external loss $(25.4 \%)$ and a poorly effective PSB trapping (front loss $35.1 \%$ ). The bad trapping originates mainly for $700 \mathrm{~nm}$ to $800 \mathrm{~nm}$ wavelength photons, which are not reflected by the PSB.

There is almost no loss below $600 \mathrm{~nm}$, which means that the dye is concentrated enough to absorb photons on two LSC thicknesses. One straightforward consequence is that reflection coefficients have to be close to unity only between 600 and $700 \mathrm{~nm}$ (after $700 \mathrm{~nm}$, photons are not absorbed by dye and will be lost at the front surface), which release the constraint on this key parameter.

Back and PLQY losses are identical even though $R_{\text {back }}=0.97$ is higher than PLQY $=0.95$, which confirms the trend of a higher sensitivity to $R_{\mathrm{back}}$ than PLQY for this case, but it cannot be generalized for all dyes, especially those with a high overlap and highly concentrated. The matrix loss is negligible because in this particular case, the photon mean traveled distance $(\bar{d})$ is around $10 \mathrm{~cm}$ and $\alpha_{\text {PMMA }}=10^{-3} \mathrm{~cm}^{-1}$ [23]. Finally, side losses are not too detrimental in this configuration because $\bar{d}$ is only two times higher than lateral dimensions.

\section{Discussion}

\subsection{Maximum concentration factor and coverage fraction}

The maximum concentration factor $c^{\max }$ has been derived by several authors [7]. It depends on the matrix refractive index $n_{\text {mat }}$ and the dye Stokes shift, and is given below:

$$
c^{\max }=n_{m a t}^{2}\left(1+\frac{\int_{E g}^{E a b s} E^{2} \exp \left(-\frac{E}{k T}\right) d E}{\int_{E a b s}^{\infty} E^{2} \exp \left(-\frac{E}{k T}\right) d E}\right)
$$

Table 2. Key values of an ideal LSC system: $c_{\mathrm{eff}}^{\max }, c_{\mathrm{int}}^{\max }$, $f 80_{\text {int }}$ and $f 80_{\text {eff. }}$

\begin{tabular}{lllll}
\hline & $c_{\text {int }}^{\max }$ & $f 80_{\text {int }}$ & $c_{\text {eff }}^{\max }$ & $f 80_{\text {eff }}$ \\
\hline No PSB & 15.0 & 0.84 & 14.4 & 0.5 \\
PSB & 4000 & $1.1 \mathrm{e}-3$ & 2900 & - \\
\hline
\end{tabular}

where $E$ is the photon energy, $E_{\text {abs }}$ is the upper cutoff energy of the dye, and $E_{\mathrm{g}}$ is the energy bandgap of the PV cell.

In our conditions, $c^{\max }$ is in good agreement $(0.35 \%$ error) with the maximum concentration factor we have found with the use of our Monte Carlo code.

The PSB increases the maximum concentration factor $c^{\max }$ by two orders of magnitude and stabilizes the optical efficiency until $f \approx 10^{-2}$.

Without PSB, the trapping depends only on total internal reflection (TIR). Thus, the PSB improves trapping by reflecting perfectly $\left(R=1\right.$ for $\lambda_{\mathrm{c}}<\lambda<\lambda_{\mathrm{g}}$ for all angles $\theta$ ) the light emitted by the dye.

To summarize these results, we work on four values extracted from Figure 5. Two of them are denoted with the subscript "int" and take into consideration only internal losses. The two other values describe the effective performance of the system (subscript "eff"), taking into account all losses. They are listed in Table 2. We define different parameters: $\quad c_{\text {eff }}^{\max }=\frac{N_{\text {coll }}}{N_{\text {tot }}}$ and $\quad c_{\text {int }}^{\max }=\frac{N_{\text {coll }}}{\left(N_{\text {tot }}-N_{\text {ext }}\right)}$, where $c^{\max }$ is the maximum concentration factor and $N_{\text {ext }}$ is the number of photons lost before entering the system, $f 80_{\text {eff }}$ is the minimal coverage fraction needed to have an effective optical efficiency of $80 \%$, and $880_{\text {int }}$ is the minimal coverage fraction needed to reach an optical efficiency of $80 \%$ of $\eta_{\text {opt }}(f=1)$.

Without PSB, these results show that losses have more impact toward the high efficiencies. In the PSB case, there is no coverage fraction enabling an effective optical efficiency of $80 \%$ because maximum efficiency is of $72 \%$ (Fig. 5) but this limiting value is already closely reached at a coverage fraction of $10^{-2}$.

\subsection{LSC robustness}

We now investigate non-ideality influence on the system performances. We use the ideal dye with non-ideal LSC parameters. We restrict ourselves at studying only the LSC used with a PSB, because performances are too low without PSB.

To quantify LSC robustness, we will focus on the value of some LSC parameters required to reduce and $f 80_{\text {int }}$ by 50,90 , and $99 \%$.

LSCs performances will be tested varying the reflection coefficient of the back reflector and also the PSB reflection coefficient (for $\lambda_{\mathrm{c}}<\lambda<\lambda_{\mathrm{g}}$ ). We will also test the nonradiative de-excitation losses through PLQY.

As shown in Figure 10, the two most critical parameters are $R_{\text {back }}$ and $R_{\mathrm{PSB}}$. They are involved in the trapping process and thus are highly correlated to the internal optical efficiency. This sensitivity can be explained remembering that the average number of reflections must 

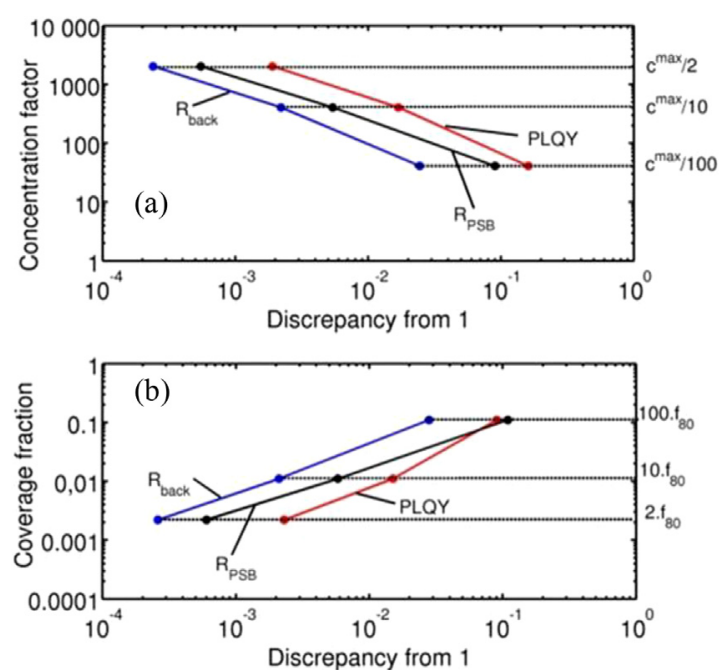

Fig. 10. Influence of PLQY, $R_{\text {back }}$, and $R_{\mathrm{PSB}}$ on $c^{\max }$ (a) and $f 80_{\text {int }}$ (b). The $x$-axis represents the discrepancy from ideality: $1-R_{\text {back }}$ (back reflector absorption coefficient) in blue, 1-PLQY (probability of non-radiative de-excitation) in red, and $1-R_{\mathrm{PSB}}$ (transmission coefficient in the high wavelength domain) in black, in the case where a PSB is used.

be on the order of $1 / f$ before reaching a PV cell if there is no loss caused. Consequently, the non-ideality consequences of $R_{\mathrm{PSB}}$ and $R_{\mathrm{back}}$ are considerable: $1-R^{1 / f}$ falls off quickly with high $1 / f$, even for almost ideal $R$.

One should insist on the fact that degradation in the range of 0.001 of crucial parameters alters the system performances by more than $50 \%$ (at $f=2 \times 10^{-3}$ ). That contributes to explain why state-of-the-art LSC systems are far from theoretical limits and why it will be difficult to approach these.

\subsection{Interdependencies of non-idealities}

A LSC is a system that exhibits highly correlated parameters. An intuitive example is the correlation between the PLQY and the dye spectral overlap. The overlap between absorption and emission spectra rules the probability of self-absorption, which will enhance nonradiative losses if the PLQY is not ideal. Thus, the sensitivity on the PLQY will be higher with a greater overlap. More generally, all parameters are correlated together, even if they seem independent. As an example, the interdependency between $R_{\text {back }}$ and PLQY is presented in Figure 10. This is best seen at very low coverage fraction $\left(f=10^{-6}\right)$.

Figure 11 does not exhibit a diagonal symmetrical axis, meaning one loss is more damaging than the other one. The iso-loss boundary (blue curve) is upshifted with respect to the diagonal axis, meaning $R_{\text {back }}$ is more prejudicial than PLQY, which is consistent with Figure 7. Since the isoconcentration factor curves (black) are not linear (slope of exponent 0.58 ), these losses are not additive. Thus, the level of performance degradation due to non-ideal quantum yield is strongly but not linearly correlated to $R_{\text {back }}$ and reciprocally.

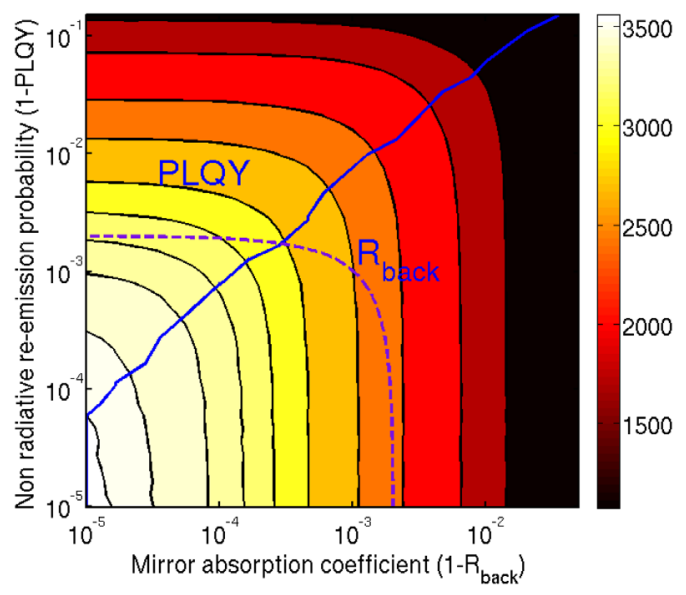

Fig. 11. Color map of the maximum concentration factor (logarithmic color scale) for different reflector absorption coefficient $\left(1-R_{\text {back }}\right)$ and non-radiative loss probabilities (1-PLQY) at $f=10^{-6}$ and $R_{\mathrm{PSB}}=1$. Black curves are isoconcentration, the blue curve separates the two domains in which the PLQY loss is higher than $R_{\text {back }}$ loss (upper domain) and reciprocally (lower domain). As a guide for the eye, the violet dashed curve represents a linear plot.

Even if there is no trivial relation between $R_{\text {back }}$ and PLQY, they are linked somehow and this link may explain the non-linear correlation.

One illustration is the example already given on overlap and the quantum yield correlations. Non-radiative de-excitation depends on the mean number of absorption $n_{\text {abs }}$, which in turn depends on the overlap: $n_{a b s} \leq 1$ if the overlap is zero, but can be higher for non-zero overlap. However, $n_{\text {abs }}$ does not depend only on the overlap. A high mean optical path (corresponding, for instance, to a low coverage fraction) will enhance the probability of selfabsorption and so will influence $n_{\text {abs }}$. This is also the case for other LSC parameters such as the back reflection coefficient. A low $R_{\text {back }}$ greatly reduces the mean optical path, which in turn reduces $n_{\text {abs, }}$, and consequently reduces the effect of a non-ideal photoluminescence quantum yield.

Reciprocally, the value of PLQY affects the system sensitivity on $R_{\text {back }}$ by the same mechanism.

The main limitation of this configuration comes from the non-optimal dye emission spectrum which almost nullified the decrease in the front loss by increasing the external loss. Indeed, the emission spectrum is broadband $(550-750 \mathrm{~nm})$ and large compared to the useful solar spectrum $(350-800 \mathrm{~nm})$.

Reflecting the emitted photons with the PSB may also mean blocking a possibly high amount of incoming useful photons (due to broadband emission). A more favorable case is to have a thinner emission spectrum to block less incoming photons while trapping them efficiently but also an emission shifted to the near infrared to increase the proportion of useful photons and also to be able to use solar cell with a lower bandgap. These should be very important conditions to be fulfilled by dyes, and an important direction for future dye design. 


\section{Conclusions}

Theoretically, concentration factors higher than X1000 are achievable with a luminescent sheet concentrator and an optical filter. The gap between theoretical limits and performances of state-of-the-art LSC relies on the very high sensitivity of non-idealities. An ideality discrepancy of 0.001 on crucial parameters can lead to a fall of $50 \%$ on the concentration factor at $f=2 \times 10^{-3}$. We have also shown that the loss mechanisms were not additives and that their respective weights depended on both physical parameters and mean basic event occurrences, making LSC a device with a strong intrication of loss channels. Thus, there are few generalities concerning loss mechanisms in LSC. The hierarchy between them has been evaluated on a case-bycase basis.

We have shown that the trends ruling the all ideal cases are valid for realistic case to a lesser extent. The realistic case shows that the main limitation comes from the dye absorption/emission spectra, and not from the PLQY (if it remains fairly high). As a consequence, LSC used with an organic dye emitting in the visible is not the best solution for LSC but can be advantageously replaced by a fluorophore emitting in the near infrared with a thinner emission width, something that could be achieved, for instance, using quantum dots with near unity quantum yield.

The authors wish to thank Gary Wolfowicz and Eric Tea for useful discussions on Monte Carlo algorithm optimization.

\section{Author contribution statement}

F.P. wrote the code, did the simulations and wrote the first version of the paper, A.-L.J. contributed to the redaction of the final version of the paper and the interpretation of the results. J.-L.P. co-supervised the work. J.-F.G. co-supervised the work and also contributed to the conception of the code, the interpretation of the results, and the final version of the paper.

\section{References}

1. W.T. Xie, Y.J. Dai, R.Z. Wang, K. Sumathy, Renew. Sustain. Energy Rev. 15, 2588 (2011)

2. G. Zubi, J.L. Bernal-Agustın, G.V. Fracastoro, Renew. Sustain. Energy Rev. 13, 2645 (2009)

3. A. Goetzberger, V. Wittwer, Sol. Cells 4, 3 (1981)

4. W.G.J.H.M. van Sark, K.W.J. Barnham, L.H. Slooff, A.J. Chatten, A. Büchtemann, A. Meyer, S.J. McCormack, R. Koole, D.J. Farrell, R. Bose, E.E. Bende, A.R. Burgers, T. Budel, J. Quilitz, M. Kennedy, T. Meyer, C. De Mello Donegá, A. Meijerink, D. Vanmaekelbergh, Opt. Express 16, 21773 (2008)
5. M.G. Debije, P.P.C. Verbunt, Adv. Energy Mater. 2, 12 (2012)

6. T. Markvart, Appl. Phys. Lett. 91, 064102 (2007)

7. G.C. Glaeser, U. Rau, Collection and conversion properties of photovoltaic fluorescent collectors with photonic band stop filters, in Proceedings of the SPIE 6197, Photonics for Solar Energy Systems, 61970L, 12 May 2006

8. L.H. Slooff, E.E. Bende, A.R. Burgers, T. Budel, M. Pravettoni, R.P. Kenny, E.D. Dunlop, A. Büchtemann, Phys. Status Solidi 2, 257 (2008)

9. V. Petrova-Koch, A. Goetzberger (eds.) High-Efficient Low-Cost Photovoltaics: Recent Developments (Springer, Heidelberg, 2008)

10. J.C. Goldschmidt, M. Peters, A. Bösch, H. Helmers, F. Dimroth, S.W. Glunz, G. Willeke, Sol. Energy Mater. Sol. Cells 93, 176 (2009)

11. A. Schüler, M. Python, M. Valle del Olmo, E. de Chambrier, Sol. Energy 81, 1159 (2007)

12. J. Bomm, A. Büchtemann, A.J. Chatten, R. Bose, D.J. Farrell, N.L.A. Chan, Y. Xiao, L.H. Slooff, T. Meyer, A. Meyer, W.G.J.H.M. van Sark, R. Koole, Sol. Energy Mater. Sol. Cells 95, 2087 (2011)

13. M.G. Hyldahl, S.T. Bailey, B.P. Wittmershaus, Sol. Energy 83, 566 (2009)

14. A.J. Chatten, D.J. Farrell, B.F. Buxton, A. Büchtemann, K.W.J. Barnham, Thermodynamic modelling of luminescent solar concentrators and modules, in Proceedings of the Twenty-First European Photovoltaic Solar Energy Conference, edited by J. Poortmans, H. Ossenbrink, E. Dunlop, P. Helm (WIP, Munich, Germany, 2006), pp. 315-319.

15. L.H. Slooff, R. Kinderman, A.R. Burgers, A. Büchtemann, R. Danz, T.B. Meyer, A.J. Chatten, D. Farrell, K.W.J. Barnham, J.A.M. van Roosmalen, The luminescent concentrator illuminated, in Proceedings of the SPIE 6197, Photonics for Solar Energy Systems, 61970K, May 12 2006

16. L. Pronneke, G.C. Glaeser, U. Rau, EPJ Photovoltaics 3, 30101 (2012)

17. P. Wurfel, J. Phys. C Solid State Phys. 15, 3967 (1982)

18. M. Peters, J.C. Goldschmidt, T. Kirchartz, B. Bläsi, Sol. Energy Mater. Sol. Cells 93, 1721 (2009)

19. M. Peters, J.C. Goldschmidt, P. Löper, B. Groß, J. Üpping, F. Dimroth, R.B. Wehrspohn, B. Bläsi, Energies 3, 171 (2010)

20. F. Proise, Study and realization of micro/nano photovoltaic cells and their concentration system, Ph.D. thesis, Université Pierre et Marie Curie - Paris VI, 2014. https://tel.archivesouvertes.fr/tel-01137589/document

21. I. Papakonstantinou, C. Tummeltshammer, Optica 2, 841 (2015)

22. Z. Krumer, W.G.J.H.M. van Sark, R.E.I. Schropp, C. de Mello Donegá, Sol. Energy Mater. Sol. Cells 167, 133 (2017)

23. S. Abrate, R. Gaudino, G. Perrone, Step-index PMMA fibers and their applications, in Current Developments in Optical Fiber Technology (Intech, 2013). http://dx.doi.org/ $10.5772 / 52746$

Cite this article as: Florian Proise, Anne-Laure Joudrier, Jean-Luc Pelouard, Jean-François Guillemoles, Loss analysis in luminescent sheet concentrators: from ideal to real system, EPJ Photovoltaics 9, 12 (2018) 